\title{
Reinstitutionalization by stealth: The Forensic Mental Health Service is the new chronic system
}

\author{
S Kaliski \\ Forensic Mental Health Services, Western Cape, Department of Psychiatry \& Mental Health, University of Cape Town, Cape Town, South Africa
}

In 1997 the Forensic Mental Health Service (FMHS) in the Western Cape had a total of 205 state patients in its database, most of whom were inpatients at Valkenberg and Lenteguer Hospitals. Currently the database has just over 800 state patients. The number of state patients in the Western Cape has more than tripled over 15 years, and there are now 9 forensic wards in the FHMS. Seven of these are low secure wards that house state patients whose abject social circumstances are the sole obstacles for granting them long leaves of absences and discharge into the community. Every month up to 4 observation cases (i.e. after being referred by the courts for assessment) are newly admitted as state patients, all of whom have serious mental illnesses (SMI). This practically results in almost 50 new state patients annually, more than enough to fill yet another ward. Discharging state patients from the provisions of the Mental Health Care Act (2002) is almost a frustratingly futile exercise as so few satisfy the stringent criteria for conditional discharge, such that about only 5 state patients can expect to be discharged annually. Practically this has created the untenable situation, in which every newly certified state patient, regardless of the nature of the charge used to admit him or her, will be institutionalized for a very long time. As has been previously pointed out, this is an egregious abuse of their human rights. ${ }^{1}$

The astonishing feature of this developing crisis is that in this era, when community and primary health care services are being developed as a priority with some ideological fervour, no one seems to have noticed that ever so quietly a burgeoning new chronic system, under the guise of caring for mentally disordered offenders, is taking us into a future that resembles the distant past. For example, a recent study on mental health policy and resource allocation in South Africa mentioned FMHS only in passing by stating that 3 (of 11 ) provinces provided 3.5 forensic beds per 100,000 population and that 3 predominantly rural provinces around Gauteng had negligible forensic services, without linking these deficiencies with those in the general system. ${ }^{2}$ There have been no systematic investigations and discussions, with consequent policy directives, on how the change in the provision of general mental health services has impacted on an almost uncontrollable expansion of the FMHS.

\section{Can we blame deinstitutionalization?}

Deinstitutionalization of psychiatric hospitals, in theory, consisted of three interlocking processes:

1. The discharge of long stay chronic psychiatric patients into the community ('dehospitalization');

2. The provision of residential care in the community;

3. Growth of community based services. ${ }^{3-5}$ In most countries the latter directive involved creating acute psychiatric units in general hospitals and clinics.

If someone needed to be admitted the assumption was, given the advances in psychopharmacology and other interventions over the last 3 decades, that this would not be for longer than a few weeks. In the developed countries most of the large tertiary care psychiatric hospitals were closed down. In 1978 in Italy Law 180 closed down all psychiatric hospitals at a single stroke. The impetus for depopulating the large psychiatric hospitals (often called 'Bins' in the UK) derived mostly from exposes on the horrific and deprived conditions long stay patients had to endure as well as the intensive ideological attacks from the antipsychiatry movement. ${ }^{5}$ This was, therefore, a purely politically motivated process, and, surprisingly, at no stage was any scientific enquiry used to determine whether, firstly, depopulating hospitals was feasible, and secondly, whether community care was indeed more humane, therapeutic and cost efficient. ${ }^{3,6}$ In some countries, such as in the UK, many of these patients were placed in halfway houses or step-down facilities (with full time staff in attendance) in the community, which despite the appearance of independent living, actually was just another form of institutionalization, sometimes called 'transintutionalization'. ${ }^{\text {? }}$

Nevertheless, the most conspicuous consequence of deinstitutionalization was the dramatic decrease in occupied beds in mental hospitals. In the USA from 1960 to 2000 the number decreased from 339 beds per 100,000 population to 21 per 100,000 population, and in Canada from 339 to 29.3,8 Between 1980 and 2008 the number of available psychiatric beds in Denmark decreased from 10,000 to 3,200.9,10 Data from South 
Africa suggests that the 23 mental hospitals in the country provide 18 beds per 100,000 population, and that these data represent decreases of up to $26 \%$ (depending on the province) over the decade before $2005 .^{2}$

As has been amply demonstrated in surveys in the wealthy developed world as well as here, community psychiatric services have either not been developed at all, have been provided erratically, or have been allocated fewer resources than needed. ${ }^{4,11}$ The result has been that significant numbers of people with SMI have ended up homeless, non compliant with their treatment, substance abusers and in prison. Penrose, in the 1930's, observed that in any society a set number of people are institutionalized at any given time, either in prisons or psychiatric hospitals, and that when the numbers in one sector decreases the numbers in the other increases. ${ }^{12}$ This has become an accepted truism. For example, when the Agnews State Hospital in Santa Clara closed in the 1970's the numbers of mentally ill prisoners in their local county jail increased 300\%. ${ }^{13}$ At least 15-20\% of prison populations contain people with SMI's, who otherwise might have been in medium or long stay wards in psychiatric hospitals. ${ }^{3}$

\section{Other consequences of deinstitutionalization}

There is now a dearth of acute beds in our general psychiatric hospitals, and those patients (now quirkily called 'mental health care users', as if they are discerning consumers) who do filter through from the community health and district hospitals generally are admitted involuntarily and not for much longer than 6 weeks. There is a constant long waiting list of 'users' for admission, which places pressure on psychiatric staff to discharge patients prematurely, just to make space for the others. Hodgins et al discovered when they compared SMI patients who had been discharged from forensic hospitals with those discharged from general psychiatric hospital that the latter were more symptomatic and scored higher on a structured risk assessment tool than the forensic patients. ${ }^{14}$

This combination of high bed occupancies due to coerced admission has been noted and ascribed to deinstitutionalization in many European countries, such as in Denmark. ${ }^{10}$ In the Western Cape the Assertive Outreach Teams have managed to prevent only a few admissions by intensively focussing on at risk patients in the community. Therefore despite the many successes of community care there will always be a significant number of chronically ill people with SMI's who cannot cope or survive outside institutional care, for whom deinstitutionalization has led to dehumanization and pauperization. ${ }^{5}$

\section{The quiet unnoticed retreat into institutional care}

A recent study in the USA's VA's 162 hospitals found that 86\% of those with SMI were reinstitutionalized either into psychiatric inpatient units, non-psychiatric inpatient units, nursing homes, or various community residential homes within 7 years of discharge. ${ }^{15}$ Similarly a comparison of service provision in 6 European countries concluded that reinstutitonalization has already begun with little debate or research, and, as with deinstitutionalization, is proceeding with without the production of systematic evidence. ${ }^{6}$ The rising numbers of forensic beds, involuntary admissions and supported housing are advanced as supposed indirect evidence of this trend.

Between 1990 and 2003 the number of beds in forensic psychiatry hospitals in England, Germany, Italy, Holland, Spain and Sweden increased from 10-143\%,5,6 The evidence that the mentally ill have increasingly been responsible for crime has been contradictory, although there have been strong indications that in some countries a much higher number of mentally disordered offenders has been noted, and that community and general psychiatric care has actually been ineffectual in preventing violent behaviour in people with SMI. ${ }^{10,14,16}$

In effect, the FMHS is being used to provide long term hospitalization and rehabilitation for people with SMI, who are violent and cannot be contained in the community. Increasingly families that cannot cope with their habitually aggressive and psychotic family members, and are exhausted by the revolving door of recurring brief admissions are being urged to lay charges in order to have that person admitted to the FMHS as a state patient for long term rehabilitation and control. Unfortunately the Law insists that all recovered state patients ultimately be reintegrated into the community in the care of their families. And it is now well known that families that do provide care and shelter for those who are aggressive are very likely to be the victims of that violence. The South African Criminal Procedure Act of 1977 was amended about 10 years ago to provide for the certification as state patients only those charged with offences that involved serious violence or other aggravating circumstances. Consequently the state patient population has become a repository of aggressive persons with SMI's. As communities become more risk averse there will be resistance to discharging many of them.

\section{The Local Reality}

At least $80 \%$ of our state patients have a history of treatment and admissions in the general psychiatric system. Colloquially they are sometimes called the 'Triple 6's', in that they have been ill for at least 6 years, have had at least 6 admissions and at least 6 different medications. Almost all derive from impoverished communities, where education levels are low, and rates of unemployment and substance abuse are high. The bed occupancy in the FMHS unit at Valkenberg Hospital is almost always over $100 \%$. When state patients are stable they are transferred to medium and low secure wards, where attempts are made to send them for gradated periods into the community in the care of their families. Although risk assessment, using the START, is used for selected patients, it is not used to determine whether any state patient should be allowed to go on leaves of absence. Otherwise there would be no space for the relentless admissions of new state patients.

A stable mental state, exemplary behaviour, good functioning in occupational therapy and a willing family generally secures a state patient the opportunity of initially enjoying days, then weekends, and then ultimately months out of the hospital. Any signs of relapse or untoward behaviour results in a call to the local police who always retrieve the hapless patient for readmission. Many have been living in the community for years, and are compliant with medication and outpatient attendance. Many state patients work in jobs during the day, and sleep in the hospital. There is an active supported employment programme run by our occupational therapists for those in the hospital and on leave in community. Over the past 20 years there have been very few incidents of serious offences committed by state patients while on leave. Admittedly many have also absconded and disappeared.

Reinstutionalization in our context, therefore, has a somewhat nuanced meaning. Most state patients are actually only partially 
institutionalized, in that they are allowed varying degrees of freedom into the community. Even those with no viable community supports are allowed to spend a day at a time to shop, attend church or work. But there is an increasing number who will remain stranded as inpatients, and almost invisible to the community and authorities, for the foreseeable future.

\section{References}

1. Kaliski SZ. Does the insanity defence lead to an abuse of human rights? Afr J Psychiatry 2012; 15:83-87.

2. Lund C, Kleintjes S, Kakuma R, Flisher AJ. Public sector mental health systems in South Africa: inter-provincial comparisons and policy implications. Soc Psychiatry Psychiatr Epidemiol 2010; 45:393-404.

3. Lamb HR, Bachrach LL. Some perspectives on deinstitutionalization. Psychiatric Services 2001; 52(8):1039-1046.

4. Sealy $P$, Whitehead PC. Forty years of deinstitutionalization of psychiatric services in Canada: An empirical assessment. Canadian Journal of Psychiatry 2004; 49(4):249-257.

5. Turner T. The history of deinstitutionalization and reinstitutionalization. Psychiatry 2004; 3(9):1-4

6. Priebe S, Badesconyi A, Fioritti A, Hansson L, Kilian R, Torres-Gonzales F et al. Reinstitutionalization in mental health care: Comparison of data on service provision from six European countries. BMJ 2005; 330:123-126.

7. Fakhoury W, Priebe S. Deinstitutionalization and reinstitutionalization: Major changes in the provision of mental healthcare. Psychiatry 2007;
6(8):313-316.

8. Chaimowitz G. The criminalization of people with mental illness. Canadian Journal of Psychiatry 2012; 57(2):1-7.

9. Kramp P, Gabrielsen G. the organization of the psychiatric srvice and criminality committed by the mentally ill. European Psychiatry 2009; 24:401-411.

10. Munk-Jorgensen P. Has deinstitutionalization gone too far? European Archives of Psychiatry and Clinical Neuroscience 1999; 249:136-143.

11. Fakhoury W, Priebe S. The process of deinstitutionalization: An international overview. Current Opinion in Psychiatry 2002; 15:187-192.

12. Penrose L. Mental illness and crime:outline of comparative study of European statistics. British Journal of Medical Psychology 1939; 18:1-15.

13. Quanbeck C, Frye M, Altshuler L. Mania and the law in California: Understanding the criminalization of the mentally ill. Am J Psychiatry 2003; 160(7):1245-1250.

14. Hodgins S, Muller-Isberner R, Allaire J. Attempting to understand the increase in the numbers of forensic beds in Europe: A multi-site study of patients in forensic and general psychiatric services. International Journal of Forensic Mental Health 2006; 5(2):173-184.

15. Irmiter C, McCarthy JF, Barry KL, Soliman S, Blow FC. Reinstitutionalization following psychiatric discharge among VA patients with serious mental illness: A national longitudinal study. Psychiatric Quarterly 2007; 78:279-286.

16. Steinart T. Reducing violence in severe mental illness. Community care does not do well. British Medical Journal 2001; 323:1080-1081.

\section{Obituary - Professor Omar Sylla}

Professor Omar Sylla was born on the 22nd of November 1948 in Dakar; he died November 5, 2012 at the age of 64, leaving a great void in psychiatry in Senegal. A Professor of Psychiatry, he received his training at the medical school of Fann Dakar under Professor Henri Collomb. He practiced psychiatry at the Moussa Diop clinic of Fann Hospital as well as in his private office. From 2002 he was President and trainer at the Senegalese Association for Systemic Family Therapy (ASTFAS) in Senegal. In 2003 he was appointed Director of the National School of Health and Social Development (ENDSS) in Dakar where he trained nurses, midwives, social workers and health managers.

Omar Sylla worked extensively on issues of migration and in 1990 he published an article in "Psychopathologie Africaine" on "Migration and Psychopathology " (Vol. XXIII 1990/1991 pp 553 / 363). As a family therapist, his research work also focused on couples from 2 nationalities. He worked closely with Genevieve Platteau, psychologist, psychotherapist and trainer in systemic family therapy with whom he coauthored two articles: "The evolution of couple in an African and a European environment; does it allow the differentiation

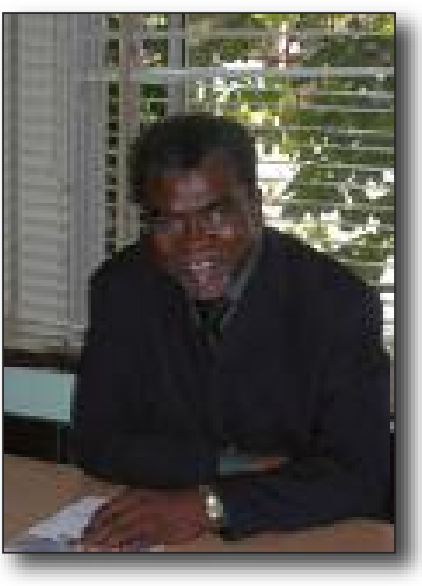

process? ": Omar Sylla \& Geneviève Platteau International Journal on systemic approaches in Geneva - Vol 29, 2008, Nº 1; "The perspectives of a contemporary couple in Europe and Africa" : Omar Sylla \& Geneviève Platteau - Review notebooks on family therapy and network practices - No. 42, 2009.

Omar Sylla was an exceptional man: he was open minded, warm, subtle, with a lot of humor; he was always optimistic even in the most painful and difficult moments of his illness. He was also a great humanist dedicated to universal psychiatry, and I had the privilege of extensively interviewing him for the first issue of Françoise Minkowska Centre's Clinical Medical Anthropology Journal "TransFaire et Cultures". Omar Sylla was a great professional who will remain present in our memory and in the world of transcultural psychiatry.

Our thoughts are with his wife Mary, his children Maimuna, Anta, Awa, Amadou, Cheick, as well with the rest of his family and colleagues.

Marie Jo Bourdin Minkowska Center, Paris, France 